\title{
Is There Still a Role for Physical Consultation in Colorectal Cancer Surveillance?
}

\author{
Hui-Yu Tham ${ }^{1} \cdot$ Jerrald Lau ${ }^{2} \cdot$ Sean Kien-Fatt Lee ${ }^{1} \cdot$ Stephen Hwang ${ }^{1} \cdot$ Dedrick Kok-Hong Chan ${ }^{1,2} \cdot$ Ker-Kan Tan ${ }^{1,2}$ (D)
}

Received: 5 June 2020 / Accepted: 8 August 2020 / Published online: 31 August 2020

(C) 2020 The Society for Surgery of the Alimentary Tract

Keywords Colorectal cancer $\cdot$ Cancer surveillance $\cdot$ Cancer recurrence $\cdot$ Physical examination

This study was conducted to evaluate how cancer recurrence was initially suspected/diagnosed for all colorectal cancer (CRC) patients from the National University Hospital, Singapore over a 5-year period, with the aim of exploring the role of community-based CRC surveillance.

After obtaining ethical approval, a review of all CRC patients without metastatic disease who underwent curative surgery from January 2012 to December 2014 was performed. All patients were followed up in the outpatient clinics in accordance to protocols adapted from the American Society of Clinical Oncology (ASCO) and National Comprehensive Cancer Network (NCCN) guidelines over 5 years. ${ }^{1,2}$ Typically, patients are reviewed 3monthly for the first 2 years and then 6-monthly for the remaining 3 years. Apart from history taking and appropriate physical examination, the surgeons or medical oncologists will go through the results of the serum carcinoembryonic antigen (CEA) levels, radiological and endoscopic investigations with the patients. Patients with early cancers are typically discharged after 5 years, while those with more advanced cancers can opt to continue surveillance in the hospital.

From a total of 507 CRC patients, 437 underwent surgery with curative intent after staging scans (Table 1). Over a median follow-up of 50 months, 81 patients $(18.54 \%)$ developed disease recurrence (Table 2). In the

Ker-Kan Tan

ker_kan_tan@nuhs.edu.sg

1 Division of Colorectal Surgery, University Surgical Cluster, National University Health System, Singapore Singapore

2 Department of Surgery, Yong Loo Lin School of Medicine, National University of Singapore, Singapore Singapore remaining 356 patients, $38(8.70 \%)$ were lost to followup, $49(11.21 \%)$ died with no documentation of disease recurrence, and $268(61.33 \%)$ remained disease-free. In these 81 patients, the index modality of suspicion was a raised CEA ( $n=24,29.63 \%)$, surveillance CT scans with normal CEA levels $(n=29,35.80 \%)$, both elevated serum CEA levels and abnormal CT scans concurrently $(n=13$, $16.05 \%)$. Twelve $(14.81 \%)$ other patients presented with non-specific symptoms. Only two $(2.47 \%)$ patients had asymptomatic recurrence diagnosed on physical examination, one of them at 7 years from the index operation.

Within the 5-year period, $436(99.77 \%)$ patients were physically assessed with no clear benefit in oncological outcomes. CRC recurrences were primarily asymptomatic and/or detected objectively via CEA and radiological scans. This echoed studies demonstrating poor sensitivity of physical examination in detection of recurrence. ${ }^{3,4}$ These findings question the advocacy of routine physical examination in guidelines for CRC surveillance postresection.

Patients with early-stage CRC often recover well, with early resumption of activities of daily living. The yield of a physical consultation is likely limited. CEA and CT scans can be performed in dedicated community health facilities, and the results traced and relayed via teleconsultation to ensure that the patients remain asymptomatic, thereby minimizing unnecessary visits to the hospitals.

Dedicated oncology-trained general practitioners can assist by overseeing the cancer surveillance alongside the management of their various other medical conditions. The existing trust and rapport between patients and their regular providers will likely improve care access, and facilitate care coordination across disciplines. ${ }^{5}$ Studies comparing CRC surveillance outcomes between GPs and specialists showed comparable outcomes in 
Table 1 Description of final sample and patients with recurrence

\begin{tabular}{lll}
\hline Characteristics & Final sample $(n=437)$ & Recurrence $(n=81)$ \\
\hline Duration of follow-up (in months), median (IQR) & $50(\mathrm{IQR}=26,62)$ & $38(\mathrm{IQR}=22,62)$ \\
Age at diagnosis (in years), median (IQR) & $65(\mathrm{IQR}=57,74)$ & $67(\mathrm{IQR}=58,76)$ \\
Gender, $n(\%)$ & $228(44.97)$ & $36(44.44)$ \\
Male & & \\
Stage of disease, $n(\%)$ & $55(12.59)$ & $5(9.09)$ \\
Stage I & $194(44.39)$ & $30(15.46)$ \\
Stage II & $186(42.56)$ & $46(24.73)$ \\
Stage III & & $5\left(6.17^{\mathrm{a}}\right)$ \\
Outcomes, $n(\%)$ & - & $69\left(85.19^{\mathrm{a}}\right)$ \\
Local recurrence & - & $7\left(8.64^{\mathrm{a}}\right)$ \\
Distant recurrence & - & - \\
Local and distant recurrence & $1(0.23)$ & - \\
Metachronous lesion, $n(\%)$ & $270(61.78)$ & $14\left(17.28^{\mathrm{a}}\right)$ \\
Disease-free, $n(\%)$ & $38(8.70)$ & $58\left(71.60^{\mathrm{a}}\right)$ \\
Lost to follow-up, $n(\%)$ & $49(11.21)$ & \\
Mortality, $n(\%)$ &
\end{tabular}

${ }^{\text {a }}$ Calculated as a proportion of patients with recurrence $(n=81)$ quality of life, disease recurrence rates, and time to detection of recurrence. ${ }^{6}$

Nonetheless, there is still a role for a physical consultation with the specialists or oncology-trained advanced nurse practitioners (APNs). Patients who undergo adjuvant therapy or proctectomy often express symptoms that may require active treatment, or simply a listening ear even if no solution is available to alleviate the concerns.

Regardless, much work is required before any overhaul of CRC surveillance. Although outcomes may be comparable between GPs and oncologists, patient acceptability and adherence to these alternatives must be evaluated. Infrastructural or logistical factors, such as existing models of care, finances,

Table 2 Disease recurrence and overall modality of disease detection

\begin{tabular}{ll}
\hline Mode of investigation & Recurrence $(n=81)$ \\
\hline CEA, $n(\%)$ & $24(29.63)$ \\
CT,$n(\%)$ & $29(35.80)$ \\
CEA and CT,$n(\%)$ & $13(16.05)$ \\
Endoscopy , $n(\%)$ & $1(1.23)$ \\
Symptoms , $n(\%)$ & $12(14.81)$ \\
Chronic cough & 3 \\
Abdominal pain & 5 \\
Symptomatic anemia & 1 \\
New onset confusion with behavioral change & 1 \\
Per-vaginal bleed & 1 \\
Altered bowel habits & 1 \\
Sign, $n(\%)$ & $2(2.47)$ \\
\hline
\end{tabular}

and provider availability, must also be considered. The current COVID-19 pandemic is unfortunately the best opportunity to imagine and innovate.

Funding Information This study was supported by the Singapore Population Health Improvement Centre (SPHERiC) (NMRC/CG/C026/ 2017_NUHS). The funders had no role in the design; collection, analysis, or interpretation of data; writing of the manuscript; or decision to submit the manuscript for publication.

\section{Compliance with Ethical Standards}

Conflict of Interest The authors declare that they have no conflict of interest.

Ethics Approval Ethical approval to conduct this study was obtained from the National Healthcare Group's Domain Specific Review Board (NHG-DSRB 2013/00870).

\section{References}

1. Benson AB, Venook AP, Cederquist L, Chan E, Chen Y-J, Cooper HS, Deming D, Engstrom PF, Enzinger PC, Fichera A. Colon cancer, version 1.2017, NCCN clinical practice guidelines in oncology. J Natl Compr Canc Netw. 2017;15(3):370-398.

2. Meyerhardt JA, Mangu PB, Flynn PJ, Korde L, Loprinzi CL, Minsky BD, Petrelli NJ, Ryan K, Schrag DH, Wong SL. Followup care, surveillance protocol, and secondary prevention measures for survivors of colorectal cancer: American Society of Clinical Oncology clinical practice guideline endorsement. J Clin Oncol. 2013;31(35):4465-4470.

3. Graham RA, Wang S, Catalano PJ, Haller DG. Postsurgical surveillance of colon cancer: preliminary cost analysis of physician 
examination, carcinoembryonic antigen testing, chest x-ray, and colonoscopy. Ann Surg. 1998;228(1):59.

4. Buie WD, Attard J-AP. Follow-up recommendations for colon cancer. Clin Colon Rectal Surg. 2005;18(03):232-243.

5. Brandenbarg D, Roorda C, Groenhof F, Havenga K, Berger MY, De Bock GH, Berendsen AJ. Increased primary health care use in the first year after colorectal cancer diagnosis. Scand J Prim Health Care. 2014;32(2):55-61.
6. Augestad KM, Norum J, Dehof S, Aspevik R, Ringberg U, Nestvold T, Vonen B, Skrøvseth SO, Lindsetmo R-O. Cost-effectiveness and quality of life in surgeon versus general practitioner-organised colon cancer surveillance: a randomised controlled trial. BMJ Open. 2013;3(4):e002391.

Publisher's Note Springer Nature remains neutral with regard to jurisdictional claims in published maps and institutional affiliations. 